\title{
Developing a knowledge-reuse tool for automatic tolerancing in product design
}

\author{
Fabio Carmo, Milton Borsato, Vitor Souza \\ Programa de Pós-Graduaç̃ão em Engenharia Mecânica e de Materiais (PPGEM), Universidade Tecnológica Federal do Paraná - UTFPR \\ e-mails: fabio.carmo@electrolux.com; borsato@uffpr.edu.br; vsouza.uffpr@gmail.com
}

\begin{abstract}
Some activities in the Product Development Process (PDP) are repetitive and time consuming, worsen productivity. Time and cost reduction are achievable through increasing the automation level of such activities, e.g., through knowledge reuse acquired on previous projects. Tolerancing is among these activities where the lack of a systematized, automatic process, leads to rework and productivity loss; there is a need to improve the efficiency of this process, based on knowledge from previous projects. Research has been performed with this objective, but normally resulting in sophisticated processes and tools, hard to implement in less developed industries. This research proposes to fill this gap, by developing a tool to support designers during the tolerancing activity, by automatically indicating tolerances for the dimensions the designer wishes to determine, in the context of an industry that designs and produces laundry machines. This challenge was approached following Knowledge-based Engineering and Case Based Reasoning principles. The evaluation phase revealed that the usage of the tool by the designers resulted in a decrease of activity time of execution and improved the performance of tolerance definition.
\end{abstract}

Keywords: knowledge reuse, geometric and dimensional tolerancing, product development process.

\section{Introduction}

Implementing knowledge reuse during the Product Development Process (PDP) can be very challenging due to three main reasons: (i) the PDP generates a massive amount of data and information, (ii) the short availability of time during work shifts makes planning knowledge management difficult and, (iii) design teams are often spread around the world, sharing different cultures and ways of interpreting data.

In an industrial context, Gao et al. (1998) states that $90 \%$ of all activities in the PDP are focused on adapting or modifying products already released, and up to $70 \%$ of the knowledge applied comes from past projects: that highlights the need for this knowledge to be properly organized, stored and retrieved.

Knowledge reuse enables creating a working environment in which repetitive tasks are executed automatically, freeing designers to focus on more creative tasks. Unfortunately, these techniques are almost entirely restricted to the Aerospace and Automotive industries (Rocca, 2011), which is intriguing since their lead times can be very long - where reducing the execution time of such tasks may not have a much relevant impact in development costs.

During the PDP, one activity that requires great deal of knowledge reuse is Tolerancing, which consists of assigning values for dimensional and geometrical variation of a specific mechanical system, limiting that variation to a range that enhances manufacturing feasibility, define product quality and assures product functionality. When specifying tolerances for product redesign or variant creation, a designer retrieves logic and standards used in previous projects. During this process, time can be saved if he could use a tool that indicates the correct values and types of dimensional and geometrical tolerances to be applied in a mechanical system.

In the tolerancing automation literature, researches propose solutions capable of automatically indicating types of tolerances. Zhong et al. (2013) propose an ontology based tool for automatic generation of assembly tolerances. Qin et al. (2015) extend the work of Zhong et al. (2013), creating an automatic geometric tolerance zones generation tool, capable of elaborating such zones graphically in a 3D assembly environment. Zhong et al. (2014) produce an assembly tolerances type model, with a logic description based approach. Sarigecili et al. (2014) propose a solution to the interoperability problem between Computer Aided Tolerancing (CAT) systems, where a GD\&T information interpretation approach is presented, in STEP format files for CAD systems. Lu et al. (2015) makes a very thorough proposition, but rather complex to implement as 
it requires the preparation of CAD models and advanced manufacturing information.

Although these researches address the automation of dimensional and geometrical tolerances, it has not been identified in the literature a solution capable of generating types and values of geometric and dimensional tolerances in the same artifact, being also simple to implement. The present research aims to fill this gap, proposing a method deployed by the utilization of a tool to automatically indicate types and values of dimensional and geometrical tolerances, with ease of implementation in an industrial context. The ultimate goal of such tool is to provide a complete list of tolerances to be applied in a mechanical system, with the user sole responsibility to insert the main dimensions of the parts being designed.

This article is structured in the following order: This introduction, a background on the approached themes, the development of the solution, its evaluation and the references used in this work.

\section{Background}

In this section, some literature background is provided, starting with the problem identification in the Knowledge Reuse in PDP theme, passing through the Design Science Research (DSR) approach, Case Based Reasoning (CBR), ending in the steps for the development of a Knowledge Based Engineering (KBE) solution.

\subsection{Knowledge reuse in the product development process}

Prior to this research, a scientific process for articles selection was performed, in order to identify the most relevant works on Knowledge Reuse in PDP, as per Carmo et al. (2017). From more than 3000 papers, the 37 most relevant works were selected to form the final portfolio. In this portfolio, three papers were conceived in the Tolerancing theme: Zhong et al. (2013), Zhong et al. (2014) and Qin et al. (2015), which were detailed previously in this paper.

After the opportunity identification, a solution has been developed using methodologies from DSR, CBR and KBE as described in the next section.

\subsection{Design science research, case based reasoning and knowledge based engineering}

Design Science Research (DSR) (Peffers et al., 2007), is the methodology used for the development of this research, which consists of six steps for the Creation Science field: i) Problem identification, ii) Definition of objectives, iii) Solution development, iv) Solution demonstration, v) Solution evaluation and, iv) Communication. The first two steps were completed through the bibliographic portfolio selection, and by bibliometric and systematic analysis on such portfolio, prior to the development of the solution, as per Carmo et al. (2017). Once the opportunity was identified, the solution development stage got underway, with the tool being constructed according to the method described in the Solution Development section of this paper. After that, it was possible to go through the phases of solution demonstration and solution evaluation, both of them being detailed in appropriate sections of this paper. The last stage of the DSR, communication, is obviously being conducted by the present article. The DSR approach prescribes the following aspects to be evaluated after solution development (1) Reality fidelity (2) Completeness (3) Robustness (4) Logic consistency (5) Usability (6) Detail level. However, it was also decided to evaluate aspects of performance and precision. With the exception of these last 2 aspects, and the detail level, all other aspects were evaluated through a survey applied to engineers, which is detailed in the Evaluation Procedure section.

The tool developed in this research follows the concept of the Case Based Reasoning (CBR) approach. According to Aamodt and Plaza (1994), CBR is a problem resolution approach, based on the register of past problem solutions, in order to solve current problems. A new problem is then solved through the comparison of a past similar case, with its solution being adapted and reused to solve the current problem.

The tool development followed the steps proposed by Curran et al. (2010), for KBE solutions development. Such as: 1) Knowledge storage; 2) Normalization; 3) Organization; 4) Modeling; 5) Analysis; and 6) Delivery.

In order to fulfill the gap identified in the literature, following the concepts presented in this section, a solution was developed and applied on an industrial environment, as detailed in the next section.

\subsection{Case of application: mechanical/industrial design}

The present research was applied on an industrial context, for testing and evaluation purposes. The case of application was developed in the product development department of a home appliances manufacturer, with relevant market share.

In the tolerance specification process developed in that department, a point of ineffectiveness was identified: the tolerance specification knowledge applied on past projects are stored in their own technical drawings and tolerance chain analysis reports. When tolerancing a new project, at some point the engineer needs to retrieve this knowledge from those sources. This step is not so simple though. First the part number of a similar part must be retrieved. To do that, the engineer needs to look for the part in a Bill of Materials list (BOM). To have access to the correct BOM, it's necessary to request it to another department, time being wasted on contacting the correct person, making the request 
and waiting for the feedback. Once the requested BOM is accessible, the engineer finds the part in the list, copying its code (part number), and consulting it into a Product Lifecycle Management (PLM) system. Finally, with the part documents accessed in the PLM, it's then possible to open up the technical drawing of such part, and verify the tolerances that have been applied. The engineer may now compare the features presented in the drawing with the ones in the new part, and reuse the tolerances of the previous part with values adaptation when necessary. As it can be seen, a system that could provide all correct types and values of tolerances to be applied in a developing part, would save a lot of work time during the tolerance specification process.

After the opportunity identification, the solution development stage got underway. The tool was developed following the method described in the next section.

\section{Solution development}

The solution is to provide the following result: After user's insertion of part's general dimensions, such as total diameter and height for a cylindrical part for example, the tool must indicate all necessary tolerances to that part, with all tolerance values adapted to the part's size inserted by the user. Therefore, the tool is suitable for utilization in the detailed design phase of the PDP, for incremental design cases, when a new product is conceived through small changes and adaptations of existing products.

Figure 1 structures the research scope. The artefact is a Microsoft Excel ${ }^{\mathrm{TM}}$ tool - the choice for this software was based on the criteria of easy access, usability, and development simplicity. Before the development of such tool (which characterizes the demonstration phase in the DSR), first a tool creation method had to be defined (the dark grey rectangle), using Knowledge-based Engineering principles as described in Curran et al. (2010). The development of the tool was performed following the principles of CBR and Knowledge-reuse, and the Demonstration section describes the steps to develop the Excel tool. Finally, the Evaluation phase consists of simulating the usage of the tool in a controlled experiment performed by final users.

\subsection{Development - tool creation method}

The tool development follows (four) steps, as illustrated in Figure 2. First, the product designed is selected, which can be represented by a 3D CAD model or a 2D Drawing. Next, proper tolerances can be chosen, which leads to building a list of tolerances and identifying which will be the exceptional dimensions - that must be identified prior to the development of the tool. This decision is based in the knowledge of types and values of tolerances used in previous products designed with similar geometry.

Drawings and tolerance chain reports must be analyzed to support the design team on which types and values of tolerances should compose the list of tolerances for a certain part, and this obviously has to be done for all the parts present in the designed product.

The implementation phase begins in the Microsoft Excel with the rules being written in the software through the elaboration of IF/THEN equations. Proportions are also configured to follow the rules previously registered. The functional capabilities of these rules are tested for coherence between input data and outputs. Finally, an interface is created according to the needs of the designer, that will operate the tool to generate the tolerances.

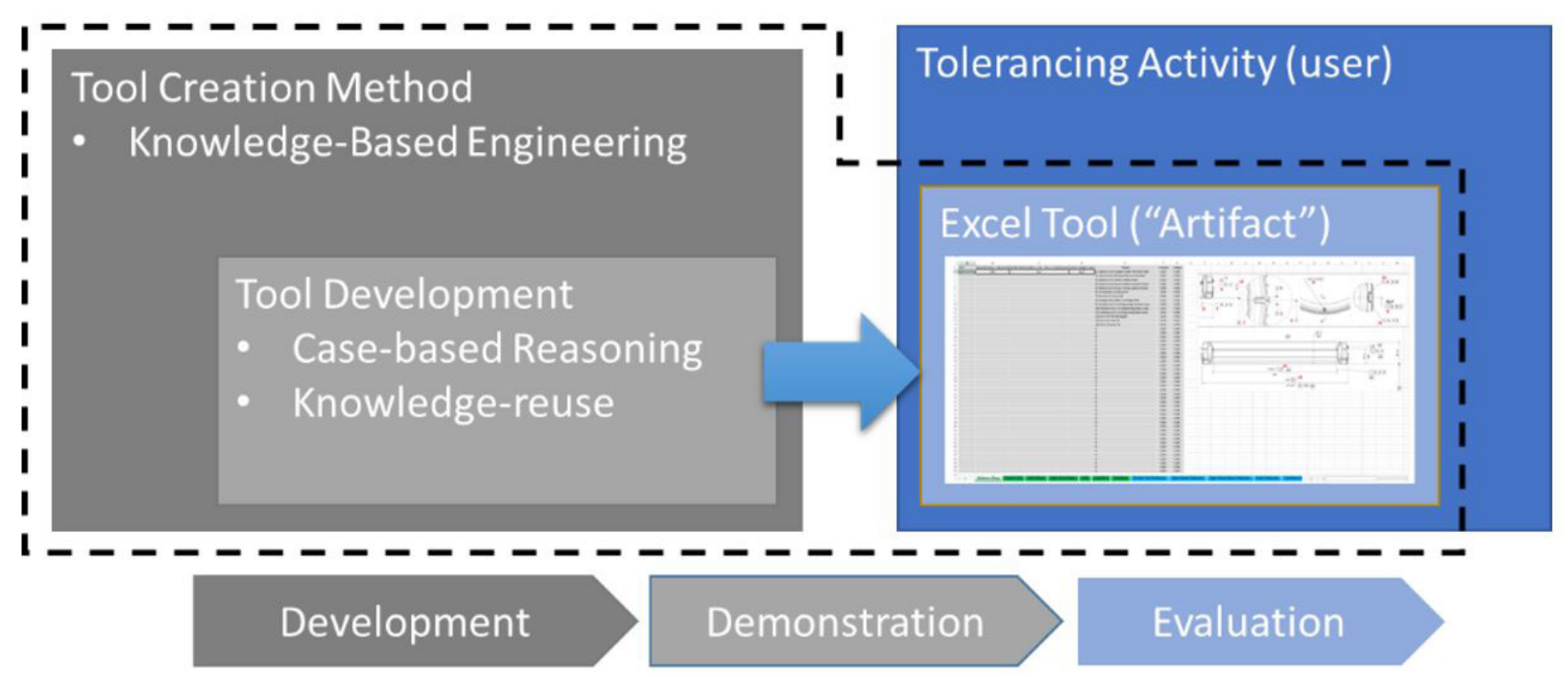

Figure 1. Research Structure. 


\subsection{Demonstration - tool development}

Based on the described method, a tool was constructed to be used in a washing machine development scenario. The numeric values of each tolerance represent the variation range that is already practiced by the company and suits manufacturing, product quality and product functionality aspects. For a part that can be found in several product models that share a certain architecture, it is reasonable to consider that it will feature minor variations in size and shape across all these products. Its general geometry can be kept for all variants; a set of tolerances that are always in the same position in the part's drawing can be defined. In this way, it becomes simple to define which tolerances should always be addressed to that part regardless of its size, and on which features those tolerances should be applied.

\subsection{Select case}

To demonstrate the deployment of the tool creation method, the example of a mechanical, circular part is used, where the whole development process can be thoroughly explained - presented in Figure 3. The main diameter, 539.9mm (numbered dimension "12"), will be the dimension to be toleranced. It must feature a linear tolerance of $+0.6 /-0.4 \mathrm{~mm}$ - carried from the knowledge base previously defined, based in the standard ISO 2768 (INTERNATIONAL..., 1989).
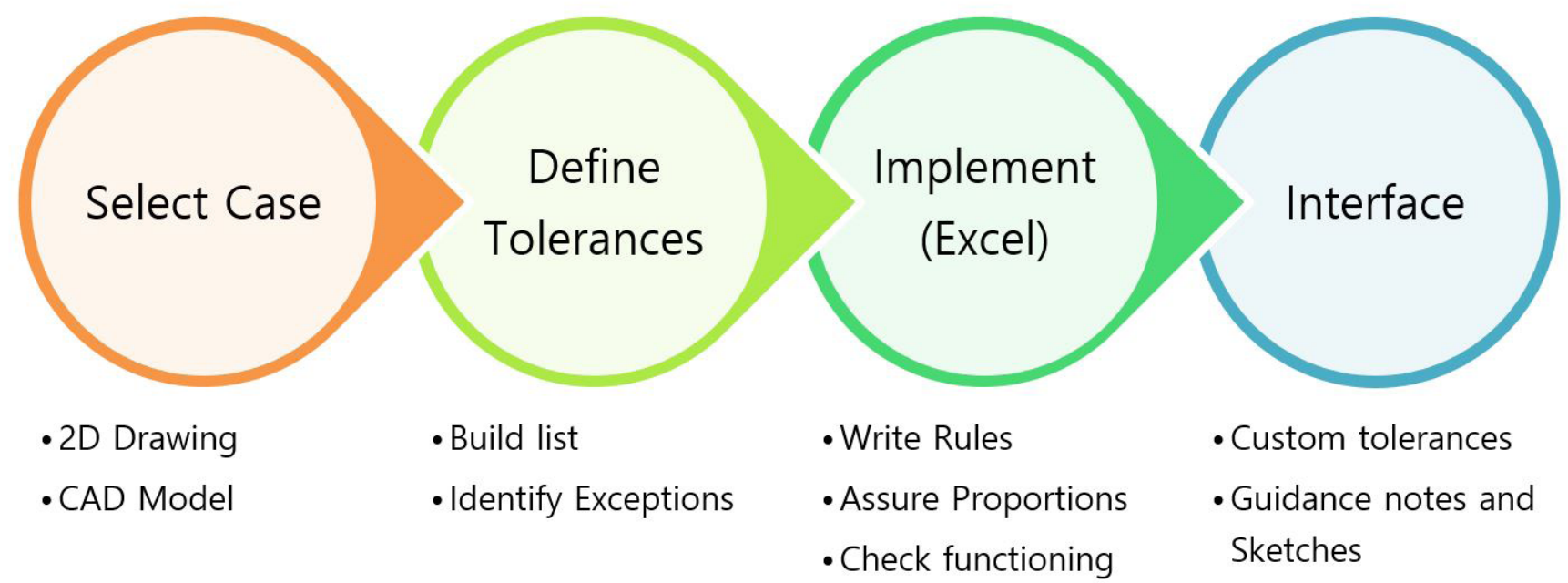

Figure 2. Tool development steps.

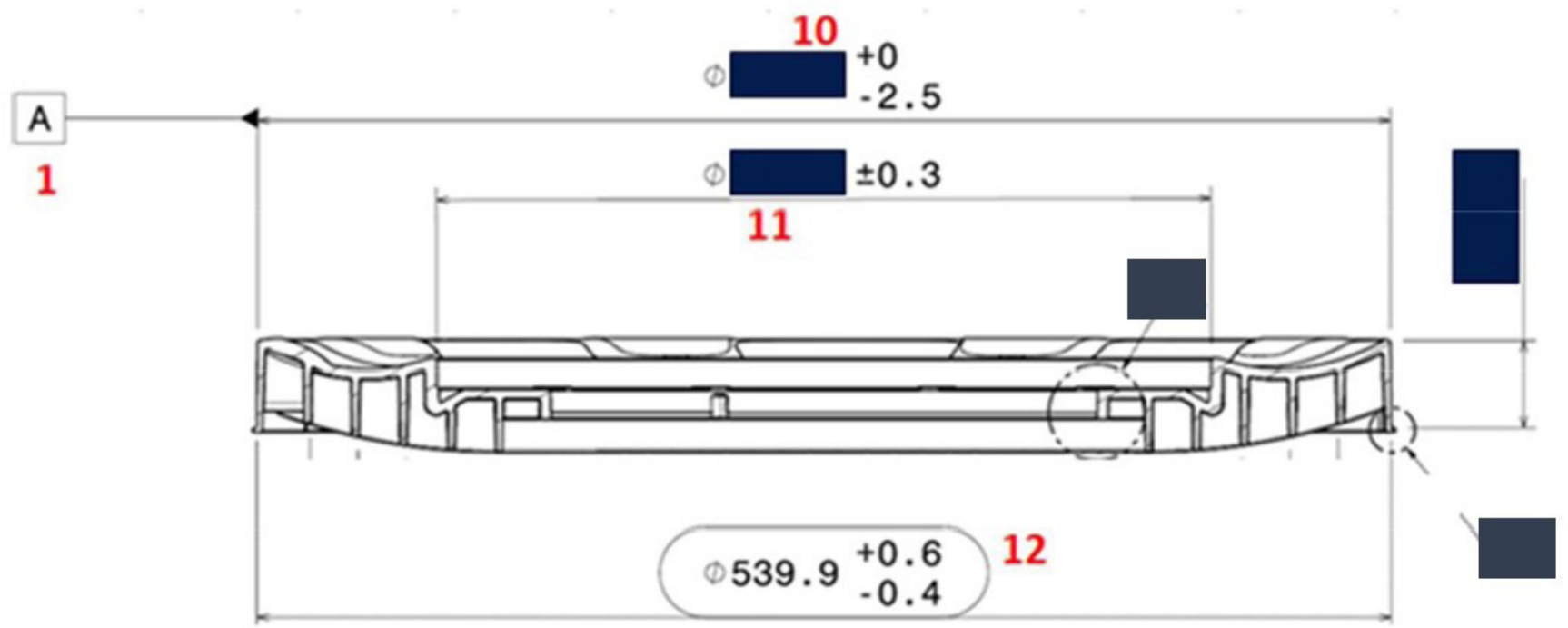

Figure 3. Example of a part that will have its tolerances generated by the artifact. 


\subsection{Defining tolerances}

The designer must assess if the part/feature related to that tolerance may present considerable size variations in the future. For the current example, it's very unlikely that this outer diameter will be smaller than $120 \mathrm{~mm}$ or bigger than $2000 \mathrm{~mm}$. Therefore, tolerances are captured from the standard, for the ranges from 120 up to $400 \mathrm{~mm}$, over 400 up to $1000 \mathrm{~mm}$, and between 1000 and $2000 \mathrm{~mm}$, as can it be seen in the lower table in Figure 4. This defines two separators to be registered in the tool (upper row in Figure 4): 400 and $1000 \mathrm{~mm}$. The tolerance in the example relates to a diameter of $539.9 \mathrm{~mm}$ of size, which has an indication of a tolerance value of $\pm 0.8 \mathrm{~mm}$ in the corresponding size range, according to the table extracted from the norm, in Figure 4. Inside the company, it was decided to use different values from the standard: $+0.6 /-0.4 \mathrm{~mm}$. It is important that the proportion among values in size ranges in the standard are respected.

In the upper part of Figure 4, it's possible to note which values of tolerances were considered for each one of the size ranges in the tool. The first size range $(0 \mathrm{~mm}$ up to $400 \mathrm{~mm}$ ) is represented by the columns named "+Result 1 " and "-Result 1 ". The second size range (over $400 \mathrm{~mm}$ up to $1000 \mathrm{~mm}$ ) is represented by the columns named "+Result 2 " and "-Result 2", while the third size range (over $1000 \mathrm{~mm}$ ) is represented by the columns named "+Result 3" and "Result 3".

The upper tolerance bounds for the higher dimensions range (+Result $3=0.90 \mathrm{~mm}$ ) is 1.5 times greater than the tolerance for the intermediate range ( + Result $2=0.60 \mathrm{~mm}$ ), respecting the same proportion in the standard, as $1.2 \mathrm{~mm}$ (medium tolerance for higher range) is 1.5 times greater than $0.8 \mathrm{~mm}$ - medium tolerance for the medium range. Following that logic, values available for all size ranges are created for this tolerance.

\subsection{Writing rules in Excel sheet}

Next, these data are consolidated in a sheet, where the feature is described, its values are declared along with the separators related to the part size, as indicated in Figure 5 for the dimension " 12 ".

It's necessary to start the creation of the mechanism that will be responsible for the selection of the correct tolerance value, to be shown for the user. The mechanism that will perform that is a value selector equation, to be inserted in the cell that will later export the selected value to be shown to the user. Equations must be constructed according to the following basic structure, which variates according to the number of separator dimensions. In this example, at most 3 separators are considered: the range with a single separator (Equation 1),

$$
I F((\$ C \$ 2>\$ D 7) ; I 7 ; G 7)
$$

Where "IF" is the logic operator indicating condition, "\$C \$2" is the imported dimensional value that has been inserted by the user, " $\$ \mathrm{D} 7$ " is a separator dimension, "I7" and "G7" are tolerance values. The meaning for the single separator equation is: "If the value inserted by the user is higher than \$D7 separator, select I7 tolerance, if it isn 't, select $G 7$ tolerance". Equation 2 is for two separators,

\begin{tabular}{|r|c|c|c|c|c|c|c|}
\hline Separator Dimension & + Result 1 & - Result 1 & + Result 2 & - Result 2 & + Result 3 & - Result 3 \\
\hline 400.00 & $1,000.00$ & 0.40 & 0.30 & 0.60 & 0.40 & 0.90 & 0.60 \\
\hline
\end{tabular}

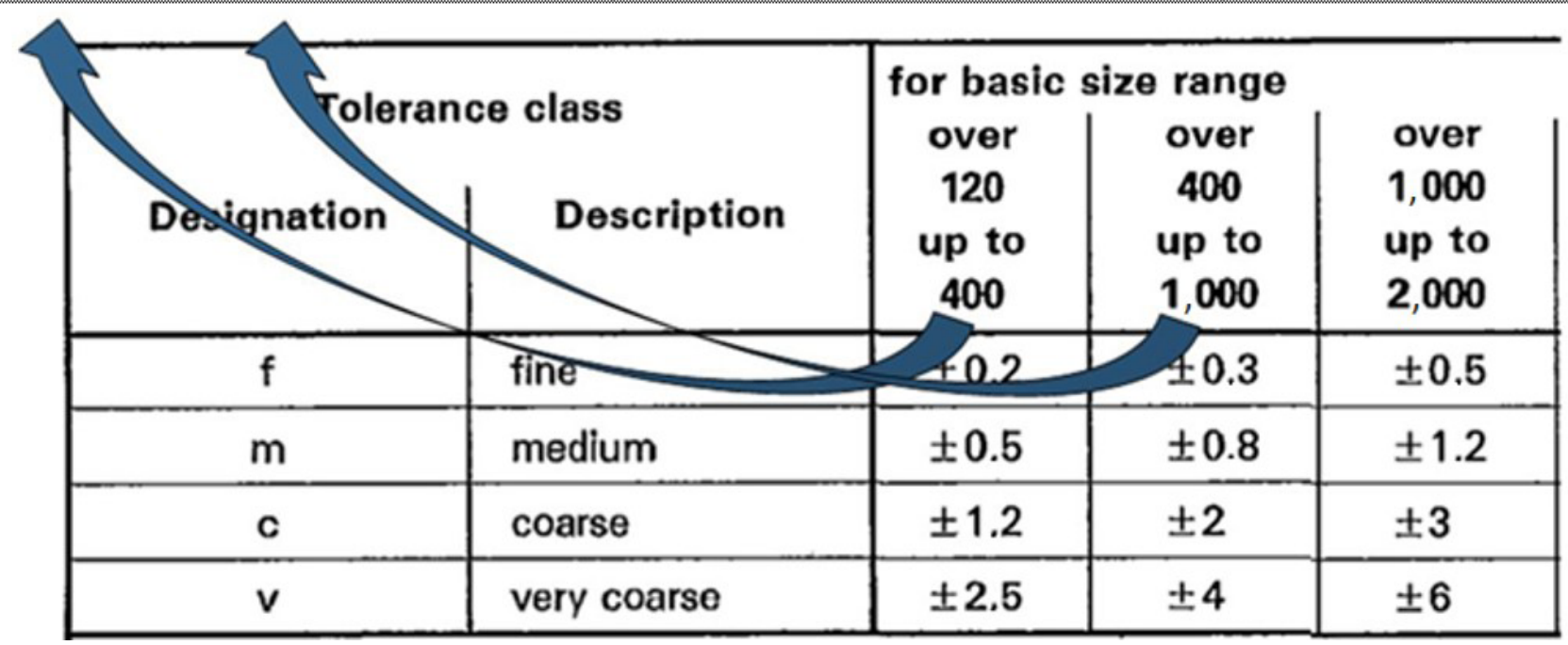

Figure 4. Separator dimensions selected for size ranges creation. 


$$
I F((\$ C \$ 2>\$ E 7) ; K 7 ; \operatorname{IF}((\$ C \$ 2>\$ D 7) ; I 7 ; G 7))
$$

Where "\$E7" and "\$D7" are the separators, and "K7", "I7" and "G7", are available tolerance values: "If the value inserted by the user is higher than \$E7 separator, select $K 7$ tolerance, if it is higher than \$D7 separator, select I7 tolerance, if it isn't, select $G 7$ tolerance". Last, Equation 3 is for three separators,

$$
\begin{aligned}
& I F((\$ C \$ 2>\$ F 7) ; M 7 ; I F((\$ C \$ 2>\$ E 7) ; \\
& K 7 ; I F((\$ C \$ 2>\$ D 7) ; I 7 ; G 7)))
\end{aligned}
$$

Where "\$F7", "\$E7" and "\$D7" are separators, "M7", "K7", "I7" and "G7" are the available tolerance values: "If the value inserted by the user is higher than \$F7 separator, select $M 7$ tolerance, if it is higher than \$E7 separator, select $K 7$ tolerance, if it is higher than $\$ D 7$ separator, select I7 tolerance, if it isn't, select $G 7$ tolerance."

In this example, the dimension input is $539.9 \mathrm{~mm}$, which is greater than the first separator $(400 \mathrm{~mm})$, but smaller than the second separator $(1000 \mathrm{~mm})$. The equation then selects the value of the second size range, which is exhibited in the tolerance positive value cell.

Once the tolerance list and the rules are ready, the user interface tab can be created for each part, showing only values requested by the user, with all the other values used in the tool being available for selection in the tab containing the selectors equations.

Figure 6 illustrates the user interface tab, where the tolerances list for a certain part is imported from the selectors equations tab.
The equations constructed are responsible for the tool's operation, and that operation is strictly related to the CBR approach. The tool must have at its disposal the most suitable tolerances values that have been used in past projects, with the equations selecting the more appropriate values for the current situation. And that is done by the comparison of the size ranges available, and the dimension that the user is inserting, just like described in the CBR methodology.

\subsection{Assuring proportions}

A mechanism to match dimensions proportions is performed, through the calculation of the dimensions of all features listed, just with an input of the total dimensions of a part, maintaining its aspect ratio across the whole geometry. This mechanism must be inserted in the cells containing the separator dimensions, as shown in Figure 7. In the example, the flatness tolerance is guided by a feature with a diameter dimension equal to $35.3 \%$ of the total diameter of the part (which is supposed to be inserted by the user). The separator dimension for the guiding feature (which is therefore $100 \mathrm{~mm}$ ), is then divided by that proportion so the separator dimensions for the total diameter is correctly presented.

When the part's total diameter input is $283.29 \mathrm{~mm}$, the guiding feature for the flatness tolerance will be $100 \mathrm{~mm}$ $-35.3 \%$ of the total diameter. The separator dimension in this case is $283.29 \mathrm{~mm}$ for the total diameter (which must be shown in the separator cell, as the user inserts the total diameter, not the dimension of the guiding feature),

\begin{tabular}{|c|c|c|c|c|c|c|}
\hline$\emptyset(\mathrm{mm})==>$ & 539.90 & 41.70 & \multicolumn{2}{|c|}{$<==$ height $(\mathrm{mm})$} & & \\
\hline Features & + Tol $(\mathrm{mm})$ & - Tol (mm) & \multicolumn{2}{|c|}{ Separator Dimension } & \multicolumn{2}{|c|}{ + Result 1 - Result 1} \\
\hline 10) Spin Bowl Docking Upper $\emptyset$ & - & 2.50 & 300.70 & & - & 1.50 \\
\hline 11) Aaitater Dacking $\alpha$ & م-20 & 年 & 555.56 & $1,388.89$ & 0.30 & 0.30 \\
\hline 12) Spin Bowl Docking Lower $\emptyset$ & 0.60 & 0.40 & 400.00 & $1,000.00$ & 0.40 & 0.30 \\
\hline 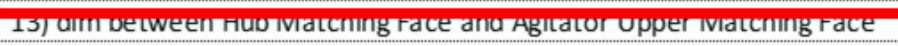 & 0.50 & 0.80 & 44.78 & 179.10 & 0.30 & 0.80 \\
\hline 14) height between Bump and Bottom & 0.50 & 0.80 & 26.90 & 116.90 & 0.30 & 0.50 \\
\hline
\end{tabular}
and $100 \mathrm{~mm}$ for the guiding feature, which is used in the mechanism operation.

\begin{tabular}{|c|c|c|c|c|c|}
\hline Part & $\varnothing(\mathrm{mm})$ & Height (mm) & Tolset & + Value & - Value \\
\hline \multirow[t]{7}{*}{ Spin Bowl Base } & 539.9 & 41.7 & 1) Datum A on Spin Bowl Docking Upper $\varnothing$ Axis & 0.00 & 0.00 \\
\hline & & & 2) Datum B on Hub Matching Face & 0.00 & 0.00 \\
\hline & & & 10) Spin Bowl Docking Upper $\varnothing$ & 0.00 & 2.50 \\
\hline & & & 11) Agitator Docking $\emptyset$ & 0.30 & 0.30 \\
\hline & & & 12) Spin Bowl Docking Lower $\emptyset$ & 0.60 & 0.40 \\
\hline & & & 13) dim between Hub Matching Face and Agitator Upper Matching Face & 0.30 & 0.80 \\
\hline & & & 14) height between Bump and Bottom & 0.50 & 0.80 \\
\hline
\end{tabular}

Figure 5. Tolerance number 12 inserted in the values generation tab.

Figure 6. User interface tab, with tolerance number 12 highlighted. 


\subsection{Check functioning}

The check functioning phase consists on the verification of the correct separation among all size ranges, as well as the correct selection of all tolerance values available in the tool. That is done by the insertion of extreme dimensional values for each one of the size ranges of each tolerance. For the case of application, it was verified that the tool worked correctly for both aspects, making it possible to move for the next phases of the development.

\subsection{Registering exceptions}

Every mechanical product has exceptions on the aspect ratio conservation among its dimensions, what leads to a necessity of deciding which dimensions must be proportional to part's total size, which dimensions must have a fixed value, and which dimensions must have a fixed difference for the total dimensions. Those decisions must be taken while building the list of tolerances for the tool. An example of that exception is given in Figure 8, where a washing machine tub is shown. In this case, the diameter formed by the suspension dockings stays at a fixed distance from the part's main diameter for all different product sizes, which is represented in the cell's separator dimension.

\subsection{Interface}

Lastly, guidance notes are registered in the tool for each tolerance. As illustrated in Figure 9, in the demonstration case conducted in this research, three columns were used for note exhibition (1) Rule category (2) Tolerance main reasons (3) Current rule. The first one informs if the tolerance

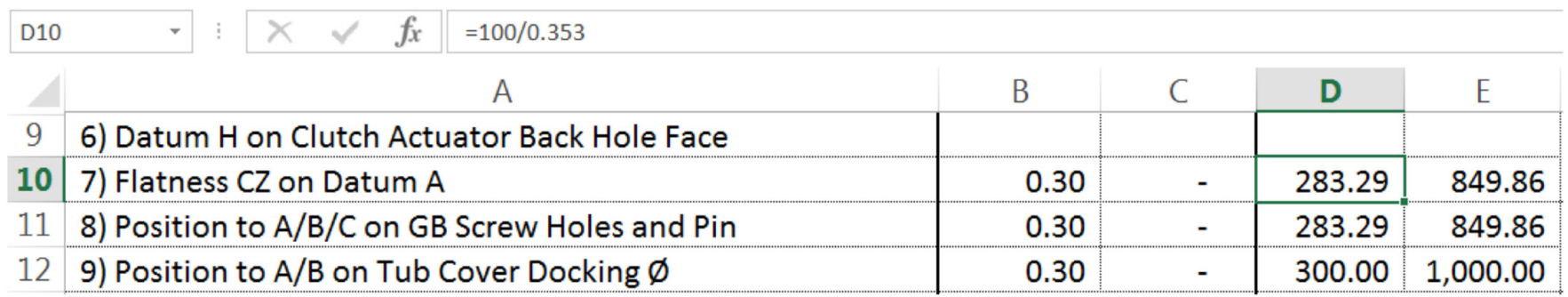

Figure 7. Aspect ratio conservation mechanism.

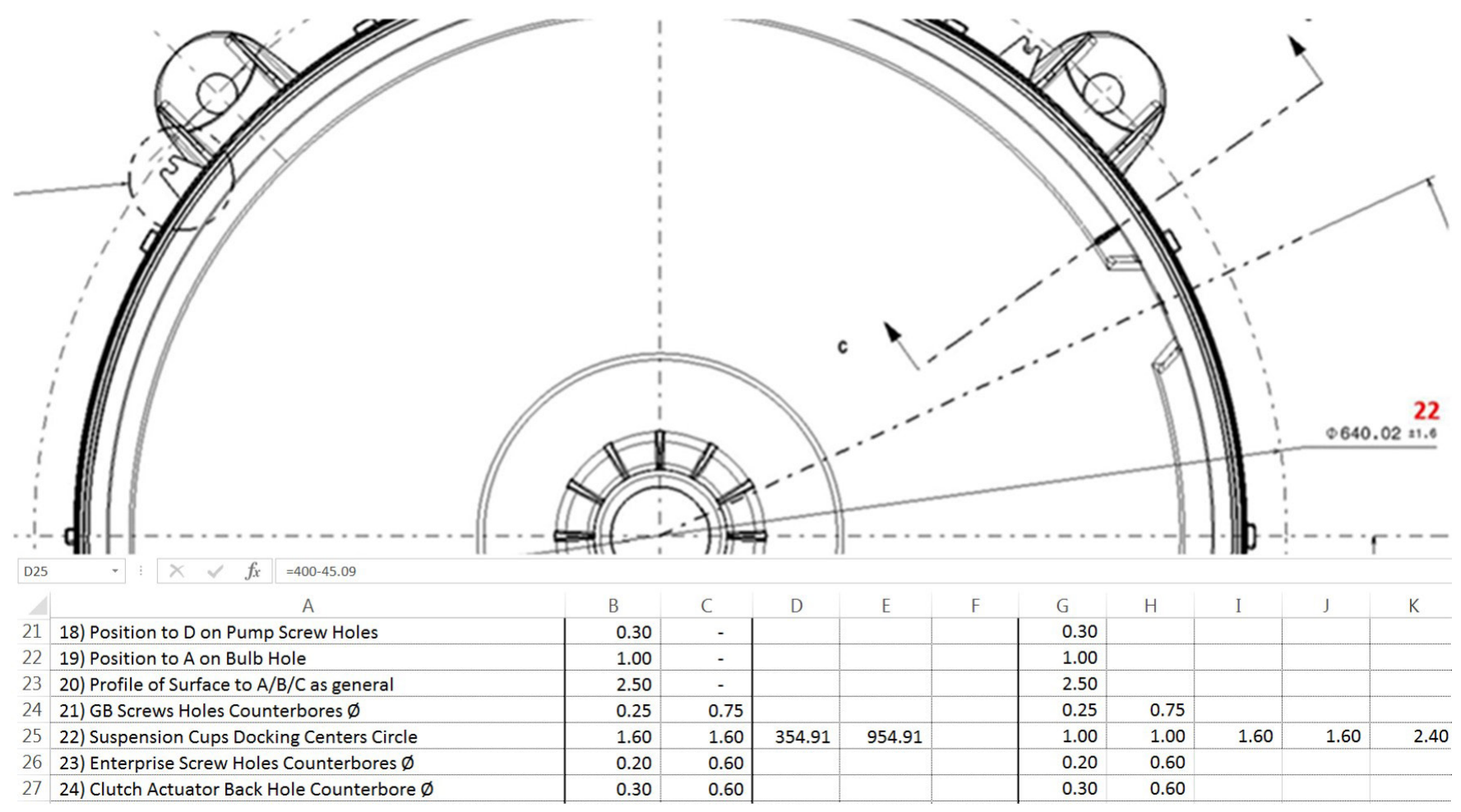

Figure 8. Exception on aspect ratio conservation mechanism. 
follows some specific standards, or just a company's common practice. The second column explains the reasons for that tolerance to exist, while the last column describes the meaning of the equation being applied to that tolerance.

It's also recommended to add drawings illustrations in the tool, for each part in the user interface tab, to make the user visualize on which position in the part the tolerances must be applied. This tool's feature can be noted in the Appendix A. Following this method for the tool's construction, the solution will present an expandable, editable and customizable aspect, being able to be used in the PDP of many types of mechanical products. The selectors equations tabs will have editable texts, and ready to use equations, being able to be applied in other situations. All tabs will be ready to receive additional items, making adaptation for a possible new scenario easier.

\section{Evaluation - tool usage}

The evaluation phase had the objective of testing the tool's utilization method, registering its performance, precision and other aspects for further evaluation. Three are the steps for using the tool; select the tab correspondent to the part being worked, insert the dimensions of the part, and then copy all tolerances outputted by the tool to the drawing or 3D model.

\subsection{Evaluation procedure}

A situation where engineers would have to specify tolerances on a 2D drawing was reproduced. Ready for tolerances 2D drawings were created, and an identical set of drawings were distributed to 5 engineers. They were to proceed according to the following instructions (1) Start timing register (2) Access 2D drawings (3) Access the tool (4) Use the tool to populate drawings with indicated tolerances (5) Stop timing register (6) Survey answering.

The DSR approach prescribes the following aspects to be evaluated after solution development (1) Reality fidelity (2) Completeness (3) Robustness (4) Logic consistency (5) Usability (6) Detail level. Nevertheless, it was decided also to evaluate aspects of performance and precision. With the exception of the last 2 aspects cited, and the detail level, all other aspects were evaluated through the survey applied to the engineers. Figure 10 shows an illustration of that survey. It is composed by 7 questions, all of them representing at least one of the mapped aspects.

The engineers gave scores from 1 to 5 to each one of the questions. The result for each aspect evaluated is the simple average among all scores given by the engineers. For example, the usability aspect is represented by questions 4 and 5 of the survey. The resulting average of the scores given by the engineers for question 4 was 3.8, and for question 5 it was 4.4. Therefore, the resulting score for the usability aspect was 4.1 , which corresponds to $82 \%$ of the maximum possible score. Table 1 compiles all results generated by the scores given by the engineers.

The reality fidelity and completeness aspects were represented by question 1 of the survey. This aspect is related to the capacity of the tool to represent the reality of the applied scenario. Both aspects reached a score of $84 \%$ of the maximum possible one.

The logic consistency aspect corresponded to question 2 of the survey. It observes the lack of logic contradiction in the solution. This aspect scored a $60 \%$ value of the maximum possible, drawing attention of the researcher due to its relatively lower value, when compared to other aspects. One hypothesis that explains this lower result is the difficulty on the understanding of what logic consistency means in the solution, a misunderstanding that could be leaded by a lack of more appropriated or detailed explanation in the survey.

The aspect of robustness was represented by survey's question number 3. It describes the capacity of the solution to adapt to other scenarios. This aspect reached an $84 \%$ score, considering the maximum possible score.

The usability aspect relates to the easiness of use of the solution, and is evaluated by question number 4 and 5 of the survey. It has reached an $82 \%$ score out of the maximum possible score.

The detail level aspect corresponds to the capacity of the tool to replicate the same level of detail present in the company's current drawings. That aspect was evaluated by the count of the number of tolerances indicated by the tool for each part, against the count of the number of the tolerances present in the current company's drawings. It was verified that the solution replicates the current detail level present in the company's drawings.

The precision aspect evaluates the capacity of the tool on not leading the user on making mistakes. The number of tolerances indicated by the solution, in the application case, was 117 . The average number of mistakes made by the engineers during the testing phase was 8 . Therefore, each engineer had 117 different opportunities to make a mistake, but only 8 mistakes were made by each one on an average. Those mistakes include wrong tolerance positioning on drawing, or specifying wrong tolerance values in contradiction of tool's indication. Precision aspect scored a $93 \%$ of the maximum possible score, considering only 8 mistakes were made out of 117 opportunities.

The performance aspect was evaluated through the tolerance specification execution time measured, for the developed method, against the time measured with the company's current method. Unfortunately, due to resources limitation in the company, the only user that executed the 


\begin{tabular}{|c|c|c|c|}
\hline A2 & $=: x$ & $\checkmark f_{x}$ & RULES TABLE \\
\hline 2 & 0 & \multicolumn{2}{|r|}{$\mathbf{P}$} \\
\hline \multicolumn{4}{|l|}{1} \\
\hline 2 & \multicolumn{3}{|r|}{ RULES TABLE } \\
\hline 3 & Rule Category & & Tolerance Main Reasons \\
\hline 4 & Common practice & Solid $r$ & ference \\
\hline 5 & Common practice & Solid $r$ & ference \\
\hline 6 & Common practice & Solid $r$ & ference \\
\hline 7 & Common practice & Alignm & ent between SBB and Balance Ring needs to be well controlled \\
\hline 8 & Common practice & Contro & Is vertical wobble of Balance Ring during spin \\
\hline 9 & Common practice & Featur & has to provide proper docking for Balance Ring \\
\hline 10 & Common practice & Effect & on assembly line \\
\hline 11 & Common practice & Effect & on assembly line \\
\hline 12 & Common practice & Effect & on assembly line \\
\hline 13 & Common practice & Effect & on assembly line \\
\hline 14 & Common practice & Effect & on assembly line \\
\hline 15 & Common practice & Effect & on assembly line \\
\hline 16 & Common practice & It cove & rs areas that are not specified by other tolerances \\
\hline 17 & Common practice & Balanc & e Ring Docking $\varnothing$ needs to be well controlled \\
\hline 18 & Common practice & Featur & e controls vertical distance between Balance Ring and Tub Cover \\
\hline 19 & Common practice & Contro & Is fitting adjustment on SBB to Spin Bowl slot, effect on assembly line \\
\hline 20 & Common practice & Contro & Is fitting adjustment on SBB to Spin Bowl slot, effect on assembly line \\
\hline 21 & Common practice & One of & the features that controls engagement of SBB Docking \\
\hline 22 & Common practice & One of & the features that controls engagement of Balance Ring Docking \\
\hline 23 & Common practice & Effect & n part's flexural strength \\
\hline 24 & Common practice & Effect & on part's flexural strength and on assembly line \\
\hline 25 & Common practice & Effect & on assembly line \\
\hline
\end{tabular}

This datum for all Spin Bowls with similar geometry.

This datum for all Spin Bowls with similar geometry.

This datum for all Spin Bowls with similar geometry.

This tol. for all Spin Bowls with similar geometry.

If part's inner $\emptyset$ is bigger than 1,000 , use $1.05 \mathrm{~mm}$ tol., if big६

If part's inner $\varnothing$ is bigger than 1,000 , use $0.45 \mathrm{~mm}$ tol., if big€

If part's inner $\varnothing$ is bigger than 1,000 , use $0.70 \mathrm{~mm}$ tol., if big६

If part's inner $\varnothing$ is bigger than 1,000 , use $0.70 \mathrm{~mm}$ tol., if bigg

If part's inner $\emptyset$ is bigger than 1,000 , use $0.40 \mathrm{~mm}$ tol., if big̨ If part's inner $\varnothing$ is bigger than 1,000 , use $0.40 \mathrm{~mm}$ tol., if big If part's inner $\emptyset$ is bigger than 1,000 , use $0.70 \mathrm{~mm}$ tol., if bigg If part's inner $\emptyset$ is bigger than 1,000 , use $0.70 \mathrm{~mm}$ tol., if big€ This tol. for all Spin Bowls

If part's inner $\emptyset$ is bigger than 998.8 (feature's 1,000), use +1 If part's total height is bigger than 1,000 , use $+0.93 \mathrm{~mm}-0.75$ This tol. for all Spin Bowls, same slot size for all This tol. for all Spin Bowls, same slot size for all If part's total height is bigger than 1,271.19 (feature's 30 at ) If part's total height is bigger than 1,107.01 (feature's 30 at ) If part's inner $\emptyset$ is bigger than $1,018.84$ (feature's 1000 ), use If part's inner $\varnothing$ is bigger than $1,007.64$ (feature's 1,000 ), use If part's inner $\emptyset$ is bigger than 1,000 , use $+3.09 \mathrm{~mm}-0.78 \mathrm{~mm}$

Figure 9. Guidance notes.

\begin{tabular}{|l|}
\hline About Excel Tool \\
\hline 1. Does the tool fit in the context of tolerances specification of washing machines design? \\
\hline 2. Is the tool free of logic contradiction? (does it have logic consistency?) \\
\hline 3. Can the tool adapt to other scenarios? \\
\hline 4. Is it easy to use? \\
\hline 5 Is it easy to understand its operation?
\end{tabular}

Figure 10. Survey applied to the designers.

Table 1. Evaluation results compilation.

\begin{tabular}{|c|c|c|c|c|c|c|c|c|}
\hline Question & Aspect & Engineer 1 & Engineer 2 & Engineer 3 & Engineer 4 & Engineer 5 & Average & $\%$ of max \\
\hline 1 & Reality Fid./ Complet. & 4 & 4 & 5 & 4 & 4 & 4.2 & $84 \%$ \\
\hline 2 & Logic Consistency & 2 & 3 & 4 & 3 & 3 & 3 & $60 \%$ \\
\hline 3 & Robustness & 4 & 5 & 4 & 4 & 4 & 4.2 & $84 \%$ \\
\hline 4 & \multirow{2}{*}{ Usability } & 2 & 4 & 4 & 4 & 5 & 3.8 & $76 \%$ \\
\cline { 1 - 24 } & & 4 & 4 & 5 & 4 & 5 & 4.4 \\
\hline 5
\end{tabular}

tolerances specification by the company's method was the researcher. The time measured was of 5 hours and 47 minutes. All the other 5 engineers, plus the researcher, executed the tolerances specification process following the developed method. The average time reached was 2 hours and 6 minutes, a decrease of $64 \%$ of execution time considering the researcher's execution time with the company's current method.

\section{Conclusions}

As it was stated in the tolerancing automation literature analysis, there is a gap in the theme, where a solution able to provide automatic indication of types and values of dimensional and geometrical tolerances, being simple to implement, was not found. That situation confirms the innovator aspect of the present research, as it fulfills 
that gap, contributing to the tolerancing automation theme. The method is still able to decrease the tolerance specification execution time for cases of incremental design, complying with evaluation aspects such as usability, precision, fidelity and others.

The DSR approach utilization contributed to the planning and organization of the present research, making clear what were the six phases that needed to be followed towards research conclusion, and what was to be done in each one of them. Its prescriptive aspect is recommended for other researches that intend to create solutions to solve specific problems, as it's the case of the present paper.

The developed method improves the traditional tolerance specification process in several aspects, including performance and precision, due its capability of knowledge reuse. It is flexible and can be used in the PDP of several types of mechanical products. According to the evaluation phase, the method was well perceived by the users, presenting good usability, robustness and detail level.

The research had some time execution limitations, which ended up generating a few future work recommendations. The first one is the complete implementation of the developed method in an industrial work environment, including the preparation of instruction procedure for tool's utilization. The second recommendation is to verify manufacturing costs available in a company and correlate them to the tolerance values indicated by the tool. The values indicated by the current developed method, correspond to the tolerances already in use by the company, and they are not necessarily the best options for manufacturing, quality and product's functionality aspects. The last recommendation is the evolution of the method to a completely automatic tolerance specification method, integrated with CAD systems, this way skipping the phase where the user needs to copy the indicated tolerances to the drawing or 3D model.

\section{References}

AAMODT, A.; PLAZA, E. Case-based reasoning: foundational issues, methodological variations, and system approaches. AICOM, v. 7, n. 1, p. 39-59, 1994.

CARMO, F.; SCHMIDT, J.; BORSATO, M. Uma análise bibliométrica sobre reuso do conhecimento no desenvolvimento de produto. Blucher Design Proceedings, v. 3, n. 12, p. 1-10, 2017.

CURRAN, R. et al. A multidisciplinary implementation methodology for knowledge based engineering: KNOMAD. Expert Systems with Applications, v. 37, n. 11, p. 73367350, 2010.

GAO, Y.; ZEID, I.; BARDASZ, T. Characteristics of an effective design plan system to support reuse in case-based mechanical design. Knowledge-Based Systems, v. 10, n. 6, p. 337-350, 1998.

INTERNATIONAL ORGANIZATION FOR STANDARDIZATION. ISO 2768: General tolerances, Geneva: ISO, 1989.

ROCCA, G. Knowledge based engineering techniques to support aircraft design and optimization. 2011. 339 f. Thesis (Doctorate). Delft University of Technology, Netherlands, 2011.

LU, H. et al. On the optimization of tolerance synthesis with an ontology-based approach. In: CHINESE AUTOMATION CONGRESS, 2015, Wuhan, China. Proceedings... Wuhan: IEEE, 2015. p. 7-12.

PEFFERS, K. et al. A design science research methodology for information systems research. Journal of Management Information Systems, v. 24, n. 3, p. 45-77, 2007.

QIN, Y. et al. Description logic-based automatic generation of geometric tolerance zones. International Journal of Advanced Manufacturing Technology, v. 79, n. 5, p. 1221-1237, 2015.

SARIGECILI, M.; ROY, U.; RACHURI, S. Interpreting the semantics of GD\&T specifications of a product for tolerance analysis. Computer Aided Design, v. 47, p. 72-84, 2014.

ZHONG, Y. et al. Constructing a meta-model for assembly tolerance types with a description logic based approach. Computer Aided Design, v. 48, p. 1-16, 2014.

ZHONG, Y. et al. Automatically generating assembly tolerance types with an ontology-based approach. Computer Aided Design, v. 45, n. 11, p. 1253-1275, 2013. 
Appendix A. Developed tool illustrations.

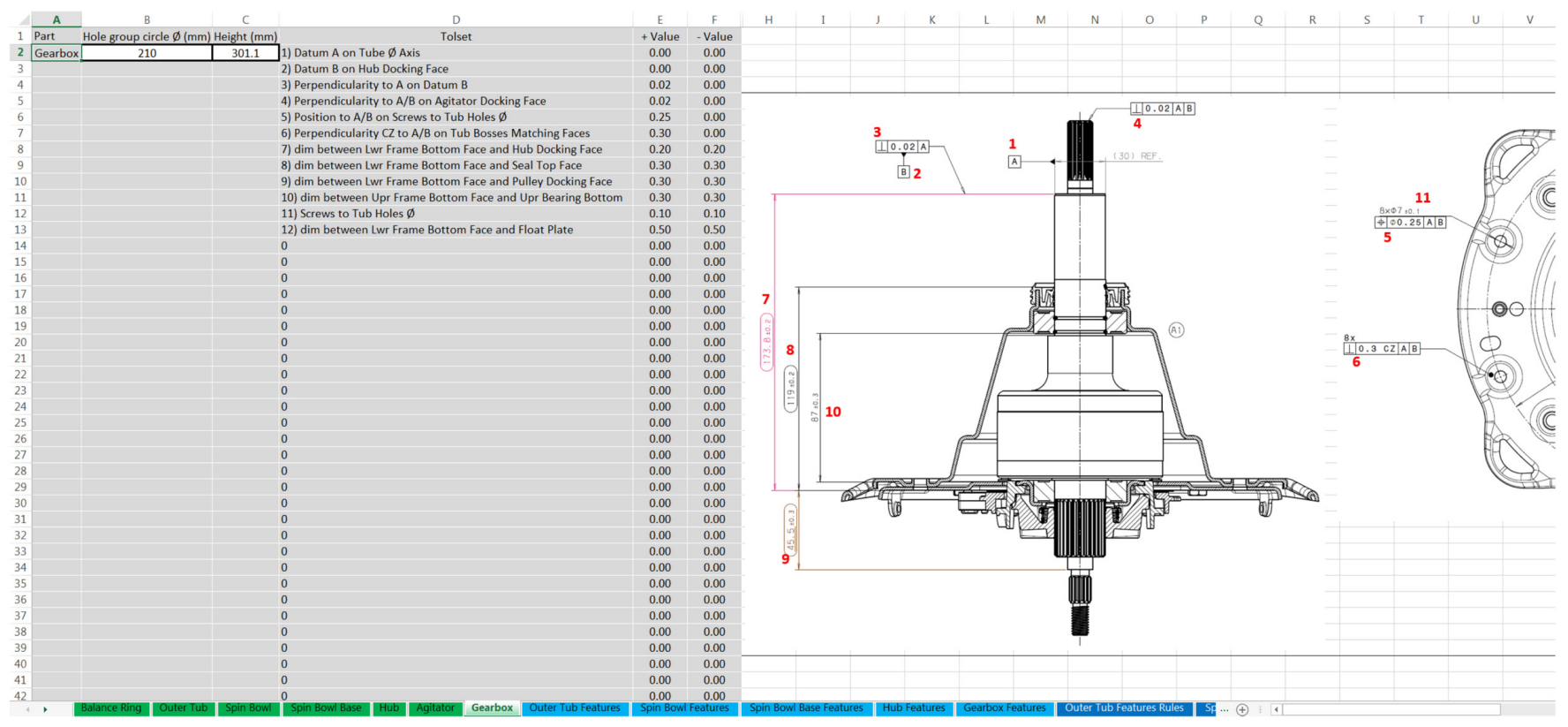

Figure A1. Tool's user interface tab.

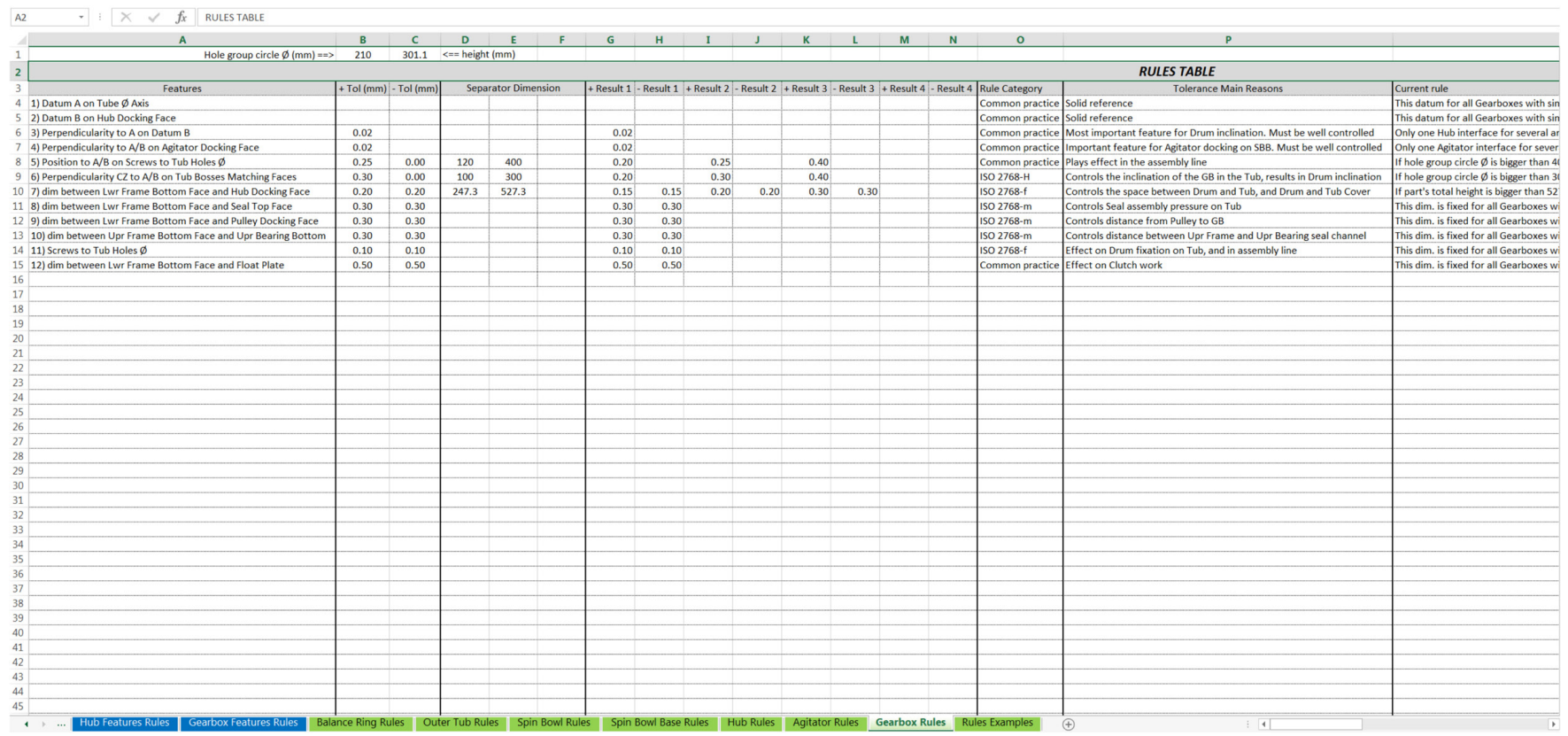

Figure A2. Tool's selectors equations tab. 